\title{
Impacto del SARS-CoV-2 (COVID-19) en la docencia teórico-práctica de la neuroanatomía humana
}

\author{
Alberto García-Barrios, Ana I. Cisneros-Gimeno, Jaime Whyte-Orozco
}

Introducción. Durante el curso 2019-2020, debido a la situación provocada por la COVID-19, Neuroanatomía humana (cuarto semestre, grado de Medicina, Universidad de Zaragoza), que en condiciones normales combina teoría y práctica presenciales, se planteó de manera telemática, mediante la aportación al alumnado de material didáctico a través de la intranet Anillo Digital Docente-Moodle ${ }^{\circledR}$ e Instagram ${ }^{\circledR}$, así como por tutorías vía Google Meet ${ }^{\odot}$.

Material y métodos. Con objeto de poder comprobar el grado de satisfacción de la docencia teórico-práctica presencial frente a la telemática, se realizaron con el alumnado dos cuestionarios (pre-COVID-19 y durante la COVID-19) de cuatro y seis preguntas cerradas, respectivamente, con cinco opciones de respuesta según una escala de Likert. Además, se plantearon dos preguntas abiertas en cada cuestionario. Por parte del profesorado, la valoración fue en una reunión de coordinación de la asignatura tras finalizar el período lectivo.

Resultados. Las encuestas de satisfacción las respondió el 71\% de los alumnos (68/95). La falta de asistencia no sufre variación significativa en ambas situaciones con respecto a la asistencia a las clases teóricas y prácticas, pero sí los motivos causantes de ausencia: los primeros fueron principalmente profesionales, y los segundos, de conectividad. El alumnado valora la docencia teórica presencial frente a la telemática de forma muy parecida, mientras que, a nivel práctico, sigue prefiriendo la presencialidad, lo que coincide con el profesorado.

Conclusión. Esta evaluación permite constatar que la docencia teórica de la asignatura por vía telemática es viable, siempre y cuando el alumnado disponga de una buena conectividad, pero no así la parte práctica, donde la presencialidad es imprescindible actualmente.

Palabras clave. Conectividad. COVID-19. Docencia. Neuroanatomía humana. Satisfacción. Vía telemática.

\section{Impact of SARS-CoV-2 (COVID-19) on the theoretical-practical teaching of human neuroanatomy}

Introduction. During the 2019-2020 academic year, and due to the situation caused by COVID-19, Human Neuroanatomy ( $4^{\text {th }}$ semester- Degree of Medicine- University of Zaragoza) which under conditions of normality combines in-person theory and practice, had to consider itself in a telematic way by providing students with teaching materials through intranet ADD (Digital Teaching Ring)- Moodle ${ }^{\odot}$, Instagram ${ }^{\odot}$, as well as tutoring via Google Meet $^{\odot}$.

Material and methods. In order to be able to check the degree of satisfaction of the theoretical teaching-face-to-face versus telematic teaching, 2 questionnaires were carried out by the students (pre-COVID-19 and during COVID-19), of 4 and 6 closed questions respectively, with 5 answer options, according to Likert scale. In addition, 2 open questions were raised in each questionnaire. On the part of the teachers, was valued in a coordination meeting of the subject, after the end of the teaching period.

Results. Satisfaction surveys were answered by $71 \%$ of students $(68 / 95)$. The lack of attendance does not suffer a significant variation in both situations, but the reasons that have led to the absence, being the first being mainly professional, and the second reasons for connectivity. Students value in-person theoretical teaching versus telematics in a very similar way, while on a practical level, they continue to prefer face-to-face, coinciding with the teachers.

Conclusion. This evaluation confirms that the theoretical teaching of the subject via telematics is viable, as long as the students have good connectivity, but not the practical part, where attendance is essential today.

Key words. Connectivity. COVID-19. Human neuroanatomy. Satisfaction. Teaching. Telematic way.
Departamento de Anatomía e Histología Humanas. Facultad de Medicina. Universidad de Zaragoza. Zaragoza, España.

Correspondencia:

Dra. Ana Isabel Cisneros Gimeno. Departamento de Anatomía e Histología Humanas. Facultad de Medicina. Universidad de Zaragoza. Domingo Miral, s/n E-50009 Zaragoza.

E-mail:

aicisner@unizar.es

Recibido:

25.03.21.

Aceptado:

30.07.21.

Conflicto de intereses: No declarado.

Competing interests: None declared.

Cómo citar este artículo: García-Barrios A, Cisneros-Gimeno A, Whyte-Orozco J. Impacto del SARS-CoV-2 (COVID-19) en la docencia teórico-práctica de la neuroanatomía humana. FEM 2021; 24: 259-62. doi: 10.33588/fem.245.1146.

(c) 2021 FEM 


\section{Introducción}

El año 2020 va a pasar a la historia por la pandemia mundial debida al virus SARS-CoV-2, causante de la COVID-19, detectada por primera vez en humanos en la provincia de Hubei (China) a mediados de noviembre de 2019 [1]. Desde entonces se fue extendiendo a otros países y el 11 de marzo de 2020 la OMS la declaró una pandemia global, dejando a su paso un gran impacto económico y social en los distintos sectores, entre ellos, el educativo [2,3]. La evolución de la pandemia provocó que, el 14 de marzo de 2020, se declarara un estado de alarma en todo el territorio nacional español y se estableció la suspensión de la actividad presencial y el cierre de las instituciones educativas en todos los niveles de enseñanza, lo que afectó prácticamente al $92 \%$ de los alumnos matriculados en el mundo (alrededor de 1.500 millones de personas) [4].

Este hecho supuso que la Universidad de Zaragoza tuviera que adaptarse de manera repentina a la nueva situación sanitaria, procediendo al cierre de sus instalaciones, y tomara una serie de decisiones en el tramo final del curso 2019-2020 para promover y flexibilizar la enseñanza teórico-práctica en línea de las asignaturas impartidas durante ese tramo de curso, así como buscar alternativas para sortear la problemática suscitada a la presencialidad, tanto a nivel de docencia como de evaluación.

\section{Material y métodos}

La asignatura de Neuroanatomía (cuarto semestre del grado de Medicina en la Universidad de Zaragoza) consta de nueve créditos ECTS y, en un curso docente habitual, su impartición conjuga la enseñanza teórica y la práctica para facilitar al alumnado una mejor comprensión de su contenido. La enseñanza teórica se realiza mediante lecciones de grupo completo en el aula (clase magistral) y debate del contenido (seminarios y tutorías). A los alumnos, antes de las clases en el aula, se les facilitan guiones que permiten su seguimiento a través del Anillo Digital Docente, intranet que utiliza la plataforma Moodle ${ }^{\odot}$ como apoyo a la docencia. La enseñanza práctica se desarrolla en la sala de disección del departamento, mediante el estudio individualizado y/o en grupo de preparaciones anatómicas (piezas naturales y maquetas), así como de cuadernos de láminas e imágenes radiológicas.

Durante el segundo semestre del curso docente 2019-2020, que dio comienzo el 10 de febrero de 2020, el desarrollo teórico-práctico de la asignatura seguía las directrices de la guía docente. Como consecuencia de la declaración del estado de alarma (14 de marzo de 2020), la actividad presencial fue suspendida, lo que afectó a la docencia teóricopráctica, que se había desarrollado sólo en un $30 \%$.

Los profesores de esta asignatura tuvieron que adaptar la metodología docente, tanto teórica como práctica, por vía telemática para poder continuar con el desarrollo del curso escolar.

Todo el material teórico-práctico pasó a estar disponible en el Anillo Digital Docente. En el caso de la teoría, en lugar de guiones, consistía en temas más desarrollados, y en el caso de la parte práctica, en láminas y ejercicios para trabajar desde casa. Instagram $^{\odot}$ y Google Meet $^{\odot}$ fueron los medios utilizados para el desarrollo de las clases y la comunicación con los alumnos.

Se ha llevado a cabo la valoración sobre este cambio metodológico debido al estado de alarma desde el punto de vista de los alumnos y desde el punto de vista del profesorado.

A nivel del alumnado, para medir el impacto de este cambio se realizaron dos encuestas (una previa al estado de alarma y otra durante éste). Estos cuestionarios (pre-COVID-19 y durante la COVID-19) han sido diseñados específicamente para el desarrollo de este trabajo, basados en una escala de Likert. Tenían cuatro y seis preguntas cerradas respectivamente, con cinco opciones de respuesta (valores entre el 1 y el 5), indicando el grado de acuerdo o desacuerdo: el 1 significaba 'muy en desacuerdo', y el 5, 'muy de acuerdo.' Además, se plantearon dos preguntas abiertas en cada cuestionario.

Se les preguntó sobre las dificultades de asistencia del alumnado a las clases teóricas y prácticas, y los motivos principales que motivaban su ausencia. También se quiso conocer el grado de satisfacción con la planificación docente tanto a nivel presencial como telemático. Las dos cuestiones abiertas fueron para conocer la opinión del alumnado sobre la realización de la actividad docente de esta asignatura únicamente por vía telemática (Tabla).

Respecto al profesorado, se mantuvo una reunión de coordinación de la asignatura al finalizar el período lectivo, vía Google Meet $^{\odot}$, para conocer la valoración personal sobre el desarrollo de la asignatura.

\section{Resultados}

Las encuestas de satisfacción las respondió el 71\% de los alumnos (68/95), lo que puede considerarse como una muestra representativa que nos permiti- 
rá sacar conclusiones válidas sobre el impacto en la docencia universitaria.

La figura 1 muestra cómo la falta de asistencia no ha sufrido una variación significativa en las clases teóricas y prácticas, ni antes ni durante la $\mathrm{CO}$ VID-19, pero sí los motivos que han propiciado ésta: los motivos profesionales eran la principal causa en un curso normal (alrededor del 50\% alumnos), mientras que los problemas de conectividad lo han sido durante las clases por vía telemática. Por otro lado, de estos resultados, podemos destacar que las ausencias debidas a motivos profesionales se reducen a prácticamente la mitad comparando los dos períodos encuestados (el 50\% pre-COVID-19 frente al 29\% durante la COVID-19), debido, en su mayoría, al confinamiento generalizado de la población. Cabe destacar en este apartado que, a pesar de que algunos de nuestros alumnos estuvieron durante este período trabajando en hospitales, bien como sanitarios, bien como voluntariado de apoyo al 061, se organizaron para, donde estuvieran, asistir a los 'directos' diarios de Instagram $^{\odot}$, algo que supuso una gran ventaja para ellos, ya que pudieron compaginar la vida laboral con los estudios universitarios.

En la figura 2 se expone el grado de satisfacción de los alumnos con la organización de las clases teóricas y prácticas, tanto presenciales como por vía telemática. Como se observa, se mantiene prácticamente estable, independientemente del tipo de docencia teórica (presencial frente a telemática) que se ha impartido a los alumnos, mientras que se observan cambios con respecto a las clases prácticas, y es menor cuando éstas se realizan por vía telemática.

Se le preguntó al alumnado si consideraba posible la realización únicamente por vía telemática de la docencia, tanto teórica como práctica. La respuesta del alumnado fue clara con respecto a la parte práctica, ya que el $74 \%$ consideró estar en desacuerdo o completamente en desacuerdo en realizarla por vía telemática, mientras que, respecto a la docencia teórica, el 53\% se mostró de acuerdo o completamente de acuerdo en realizarla únicamente por vía telemática.

Respecto al profesorado, en una reunión de coordinación de la asignatura por vía Google Meet $^{\odot}$, la valoración de los profesores fue unánime y consideraron que la docencia teórica de esta asignatura puede llevarse a cabo mediante los métodos utilizados estos meses, mientras que la docencia práctica de estas asignaturas requiere cierto grado de presencialidad para preservar el proceso de enseñanza-aprendizaje del alumno, ya que se consideró que es difícil suplir las actividades que se reali-
Figura 1. Dificultad para asistir a clase teórica y práctica, y motivo principal.

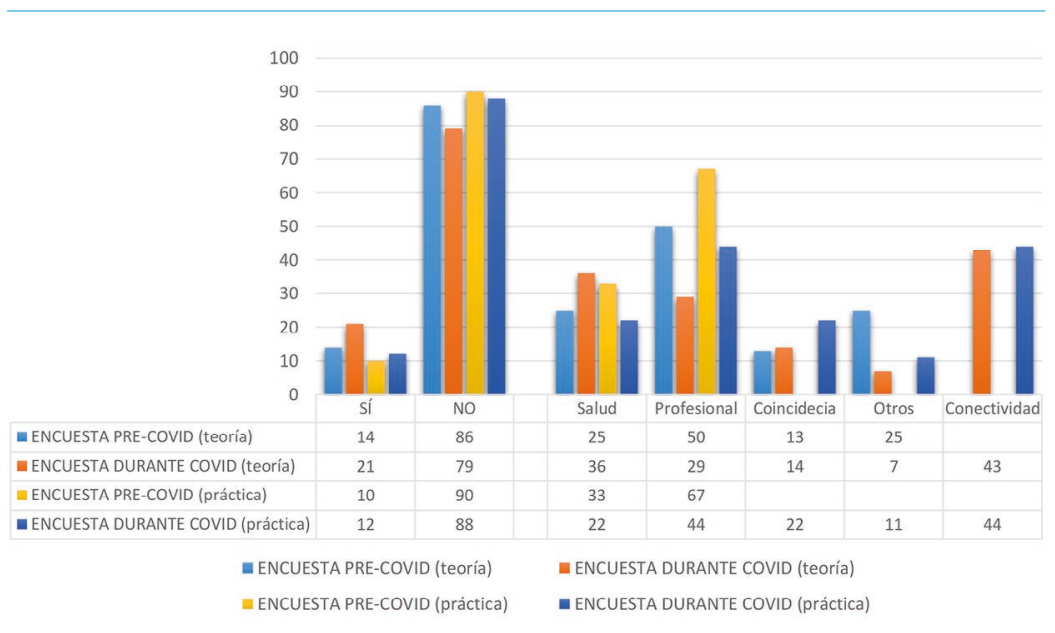

Figura 2. Grado de satisfacción con la organización de las clases teóricas/prácticas.

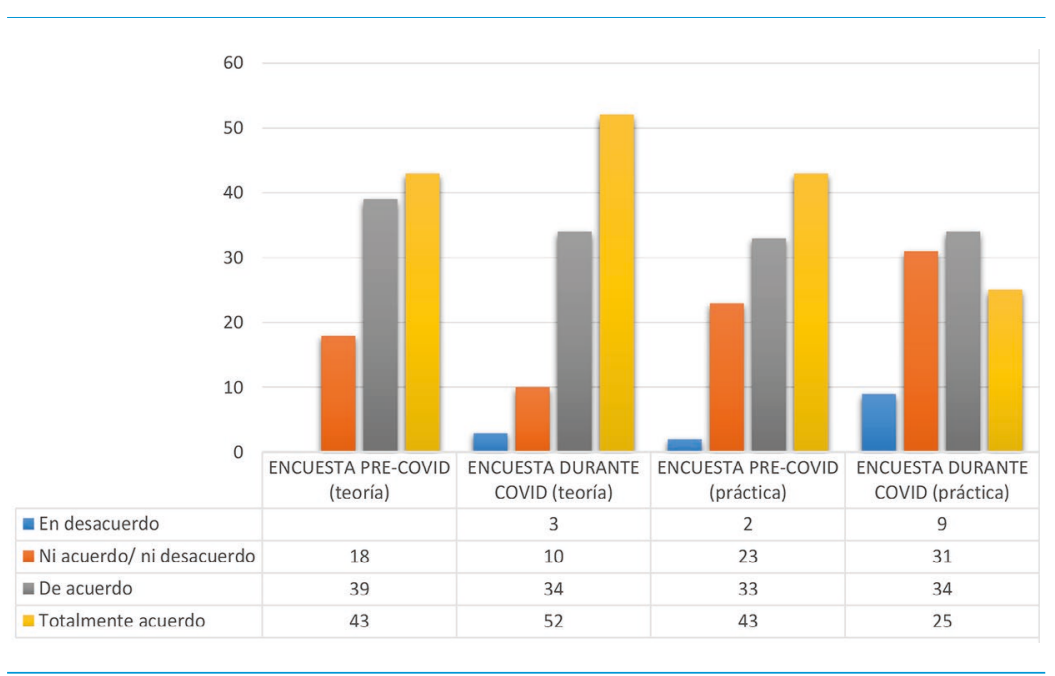

Tabla. Cuestionario planteado al alumnado.

\begin{tabular}{ll}
\hline Preguntas 1-2 & $\begin{array}{l}\text { ¿Has tenido alguna dificultad para poder asistir a las clases teóricas y prácticas } \\
\text { presenciales/telemáticas? }\end{array}$ \\
\hline Preguntas 3-4 & $\begin{array}{l}\text { ¿Cuál es el motivo por el que no has asistido a las clases teóricas y prácticas } \\
\text { presenciales/telemáticas? }\end{array}$ \\
\hline Preguntas 5-6 & $\begin{array}{l}\text { ¿Consideras correcta la planificación docente para las clases teóricas y prácticas } \\
\text { presenciales/telemáticas? }\end{array}$ \\
\hline Pregunta 7 & $\begin{array}{l}\text { ¿Consideras posible que las clases teóricas se realicen únicamente por vía } \\
\text { telemática? (Sólo cuestionario durante la COVID-19) }\end{array}$ \\
\hline Pregunta 8 & $\begin{array}{l}\text { ¿Consideras posible que las clases prácticas se realicen únicamente vía telemática? } \\
\text { (Sólo cuestionario durante la COVID-19) }\end{array}$ \\
\hline
\end{tabular}


zan en la sala de disección (cadáver, pieza anatómica natural o maqueta) únicamente con material gráfico (imágenes y/o láminas) con los medios disponibles en nuestra facultad hoy en día.

\section{Discusión}

El efecto que ha supuesto la pandemia producido por el SARS-CoV-2 ha puesto en evidencia las virtudes y deficiencias del sistema educativo, teniendo que recurrir a la tecnología para poder continuar de una manera, lo más normalizada posible, con el proceso de enseñanza-aprendizaje de los alumnos en el curso 2019-2020, desde los primeros niveles educativos hasta la universidad, y supondrá un importante cambio en la forma de afrontar el sistema educativo de aquí en adelante [5]. En el grado de Medicina, hasta en las asignaturas más clínicas, debido al cierre de facultades y hospitales, la docencia práctica se ha realizado mediante diversas tecnologías (vídeos prácticos, casos clínicos interactivos e incluso realidad virtual) y se ha mantenido el contacto con el alumno a través de videoconferencias [6].

\section{Conclusiones}

Como hemos evidenciado en nuestro estudio, la pandemia ha producido un cambio importante en la manera de impartir la docencia, que puede plantearse de manera telemática. Sin embargo, para que ésta pueda llevarse a cabo, debemos asegurar que el alumnado pueda disponer de una buena conectividad, principal causa que ha provocado la falta de asistencia a las clases tanto teóricas como prácticas durante este período.

Además, habrá que tener en cuenta los recursos de los que dispone cada centro para desarrollar la parte práctica de la docencia en Anatomía humana, donde el material gráfico, ya sea láminas o vídeos, no puede suplir lo utilizado de manera ordinaria en la sala de disección y es necesaria la inversión en modelos anatómicos en 3D y en otras nuevas tecnologías.

Bibliografía

1. Ahmed H, Allaf M, Elghazaly H. (2020). COVID-19 and medical education. Lancet Infect Dis 2020; 20: 777-8.

2. Dashraath P, Wong JLJ, Lim MXK, Lim LM, Li S, Biswas A, et al. Coronavirus disease 2019 (COVID-19) pandemic and pregnancy. Am J Obstet Gynecol 2020; 222: 521-31.

3. Mas-Coma S, Jones MK, Marty AM. COVID-19 and globalization. One Health 2020; 9: 100132.

4. Unesco. COVID-19 Impact of Education (2020). https://bit. ly/2yJW4yy. Fecha de última consulta: 12.02.2021.

5. Costa MJ, Carvalho-Filho M. A new age for medical education after COVID-19. Fundacion Educación Médica 2020; 23: 55-7.

6. Núñez-Cortes JM. Educación Médica durante la crisis por COVID-19. Educacion medica 2020; 21: 157. 